\title{
TRANSFORMATION OF AN INEERENCE TREE TO A LOGICALLY EQUIVALENT NEURAL NETWORK
}

\author{
A.Molotkov* and A.Borisov** \\ *Post Graduate Student, **Professor of Computer Science \\ Specialized Institute of Intelligent Computer Technologies \\ Technical University of Riga \\ I, Kalkyu Street, Riga Lv - 1658, Latvia \\ Phone: +3717320019 \\ Fax: +3717820094 \\ E-mail:aborisov@egle.cs.rtu.lv
}

\section{INTRODUCTION}

III-formalized situations can easily be represented by means of conditional rules. Heuristics that are represented through rules well describe the relationships among the factors of the process or event to be examined.

In such a situation two tasks should usually be solved. The first deals with constructing the logical structure of a set of rules which properly represent the described process. The second task is the adjustment of this structure on the level of quantitative evaluations which characterize the described relationships. A logically full, consistent and non-exhaustive set of rules is adjusted through learning procedures taken from the apparatus of artificial neural networks (Zurada 1992).

When implementing this approch, the following tasks arise that are considered in the present:

(i) construction of the adequate neuronlike structure,

(ii) choice and implementation of a learning algorithm for training the above structure.

Other investigations are also known that have used a neural network as an element of an expert system. Gallant (1986) proposes the 
methodology of artificial neural network application for the task of knowledge acquisition task.

\section{INITAL REQUIREMENTS TO THE REPRESENTATION OF A KNOWLEDGE BASE}

A knowledge base should meet the requirements that follow:

(1) An inference tree, on the basis of which a logical neural network is being constructed, implements a straightforward reasoning chain (straightforward inference) (Harmon and King, 1985).

(2) Presence of the consistent rule base. For example, a rule base should not contain the rules of the form:

- IF A and B THEN C

- IF $A$ and $B$ THEN not $C$.

(3) All the rules should be reduced to the following form ( $\mathrm{Li} \mathrm{Min} \mathrm{Fu}$, 1993):

$$
\text { If } \alpha_{1} \text { and } \alpha_{2} \text { and } \ldots \alpha_{n} \text { Then } \beta,
$$

where $\alpha_{1}, I=1, \ldots, \mathrm{n}$ are attributes contained in condition part of the rule, $\beta$ is the consequence of the rule.

If rules are of another format, they should be changed by means of the following equivalent transformations:

- "If $P$ Then $Q$ and $R$ " $\Leftrightarrow$ "If $P$ Then $Q$ " and "If $P$ Then $R$ "

- "If $P$ or $Q$ Then $R$ " $\Leftrightarrow$ "If $P$ Then $R$ " and "If $Q$ Then $R$ "

- "If $P$ and ( $Q$ or $R$ ) Then $S " \Leftrightarrow$ "If $P$ and $Q$ Then $S "$ and "If $P$ and $R$ Then $S "$

\section{CONSTRUCTION OF AN ADEQUATE NEURAL NETWORK}

The process of network construction is implemented through three stages.

1. Construction of a global neural network. At this stage, a neural network based on the inference tree structure, the so-called global neural network, is constructed. The number of network layers, the number of neurons in each layer, as well as the structure of relationships among separate neurons of the network are determined. 
2. Decomposition of the global neural network into local 2-level nets. At this stage, the network constructed on the basis of an inference tree is decomposed into as many 2-level networks (local neural networks) as many levels of inference the corresponding inference tree has. If the global neural net has no hidden layers, i.e. an inference tree has a single inference level, this stage is not performed. The necessity for the global neural network decomposition could be explained as follows. First, local neural networks . enable making intermediate (local) inferences which precede the main, global inference. To obtain intermediate inferences in the global neural network, is either very difficult or impossible. Second, it easier to train local networks than to train global ones.

3. Inclusion of additional hidden layers into local neural networks. Depending on the number of input neurons and the similarity of input and output patterns, one or more hidden layers are included into each local neural network. Additional hidden layers enable successful training of local neural networks.

Local logical neural networks created and trained at the preceding stages enable emulation of the inference engine of a knowledge-based system, which implements a straightforward reasoning chain. By obtaining numerical input data that are the facts about the problem domain coded in the numerical form, each local neural network should represent such numerical values in its outputs after decoding of which the same conclusions could be obtained as the ones produced by the knowledge-based system inference engine on the basis of analogous facts.

The rule base underlying logic is transformed to a neural network by means of network training. The network trained through the examples taken from the rules truth table enables implementation of any logical operation laid down in this truth table.

\section{ANALYSIS OF THE EXPERIMENTS}

To check the equivalence of the conventional inference tree based approach to the implementation of logical inference engine and the proposed, artificial neural net based approach, a number of experiments were performed.

Before the experiments are made, a logically equivalent neural net is constructed and trained. Each experiment passes through several stages:

- generation of input network values; 
- computation of output network values;

- matching of obtained values with the required values from the truth table on the basis of which the present neural network has been trained.

A set of rules proposed in (Harmon and King, 1985), known as theatre knowledge base, was used as a knowledge base in this paper.

On the basis of an inference tree, a global neural networks was constructed which was later decomposed into two local neural networks. The first local neural network that has one hidden layer of three neurons, implements logical inference equivalent to the first inference level in the inference tree. The second local neural network which has two hidden layers of nine neurons in each, implements logical inference equivalent to the second inference level in the inference tree.

For all possible combinations of attributes contained in conditional parts of rules the truth table was constructed. For each combination of attributes in the truth table a chain of local and global inferences obtained from the inference tree is shown. The truth table for the first local neural network contains 9 examples, where as the table for the second local neural network contains 24 examples.

In order to cover all possible combinations of attributes, 24 experiments were performed. In each experiment, the set output values of the 1 st and 2 nd neural networks was equivalent to the logical inference chain obtained on the basis of the inference tree.

\section{CONCLUSIONS}

The paper proposes a method for implementing a logical inference engine based on artificial neural networks. To that end, on the basis of the inference tree structure of the corresponding knowledge base, an artificial neural network is constructed that is trained by the corresponding algorithm intended for this architecture.

A number of experiments were performed to examine the equivalence of two approaches to the implementation of an inference engine, i.e. the conventional, inference tree based approach, and the proposed, neural networks based one. Experimental data have shown the efficiency of the proposed method for the implementation of artificial neural network based inference engine. 


\section{REFERENCES}

Gallant S. I. Connectionist expert systems. Communications of the ACM. Vol.31. - N2. - 1988. - 153. - 169. p.

Harmon P. and King D. Expert Systems: Artificial Intelligence in Business. J.Wiley (Ed.). - New York, 1985.

Li Min Fu, Knowledge-based connectionism for revising domain theories. IEEE Transactions on Systems, Man and Cybernetics. Vol. 23. N 1. - 1993. - 173. - 182. p.

Rumelhart D. E., Hinton G. E. and Williams R. J. Learning internal representation by error propagation. Parallel Distributed Processing. Rumelhart and McVelloud (Eds). - Vol. 1, 2. - Massachusetts: MIT Press, 1986. - 318. - 362.p.

Tan C. L., Quah T. S. and Teh H. H. An artificial neural network that models human decision making. IEEE Computer Society, 1996. - 64. 70. p.

Zurada J. M. Introduction to Artificial Neural Systems. - St.Paul: West Publishing Company, 1992. - $683 \mathrm{p}$.

Nelson M. and Illing Worth W. T. A Practical Guide to Neural Nets. Addison: Wesley Publishing Company, 1991. 